\title{
The Effect of Nutritional Education Program Based on Health Belief Model (HBM) on the Knowledge of Fasting Type 2 Diabetic Patients
}

\author{
Mehrnoosh Sadeghi Goorabi ${ }^{1}$, Mahdieh Akhoundan ${ }^{2}$, Zhaleh Shadman ${ }^{3}$, Majid Hajifaraji ${ }^{4}$, Mohsen Khoshniat Nikoo ${ }^{2}{ }^{2}$ \\ 1- International Branch, Shahid Beheshti University of Medical Sciences, Tehran, Iran. \\ 2- Diabetes Research Center, Endocrinology and Metabolism Clinical Sciences Institute, Tehran University of Medical Sciences, Tehran, Iran. \\ 3- Endocrinology and Metabolism Research Center, Endocrinology and Metabolism Clinical Sciences Institute, Tehran University of Medical Sciences, \\ Tehran, Iran. \\ 4- National Nutrition and Food Technology Research Institute, School of Nutrition Sciences and Food Technology, Shahid Beheshti University of \\ Medical Sciences, Tehran, Iran.
}

\section{A B S T R A C T}

Background and Objectives: Studies have shown that many people with diabetes are fasting in the holy month of Ramadan. Ramadan fasting causes several changes in metabolism, habit and lifestyle that are associated with an increased risk of hypoglycemia and hyperglycemia in diabetic patients. An educational program based on behavioral science theory in the field of diet and medication changes, proper physical activity during Ramadan fasting, recognizing risks and symptoms of fasting complications and the management may improve the diabetic patients' ability in changing lifestyle, reduce the risk of fasting complications and weight and metabolic control during Ramadan. This study aimed to investigate the effect of nutritional education program based on health belief model (HBM), on the knowledge of type 2 diabetic patients who fast in Ramadan.

Materials and Methods: This randomized clinical trial was conducted on 53 type 2 diabetic patients referring to the Diabetes Clinic of Endocrinology and Metabolism Research Institute, Tehran University of Medical Sciences and tending to fast during Ramadan, who were recruited based on the research inclusion criteria. The subjects were randomly assigned into two groups of intervention $(n=28)$ and control $(n=25)$. A week before Ramadan, the intervention group was educated based on HBM. In a week before and after Ramadan, the data on demography, HBM components and knowledge were collected through face-toface interview by trained nutritionists.

Results: The research results revealed that the education program in the intervention group significantly increased the HBM components such as perceived severity, perceived benefits, perceived barriers, and self-efficacy comparing to the baseline and the control group $(\mathrm{P}<0.005)$. At the end of the study, there was a significant difference in the scores of knowledge about fasting conditions and medications, nutrition and physical activity between the two groups $(\mathrm{P}<0.001, \mathrm{P}=0.049$ and $\mathrm{P}=0.013$, respectively). The educational intervention significantly increased the total knowledge score, as well as the fasting conditions and medications and blood glucose control scores compared to the baseline values; also in comparison with the control group, the scores of fasting conditions and medications, blood glucose control and total knowledge increased significantly in the intervention group.

Conclusions: The findings showed an increase the knowledge level and improvement in the HBM components in the intervention group compared with the control group. It seems that focused Ramadan education could increase Ramadan knowledge of patients and empower them to proper management of diabetes during the holy Ramadan.

Keywords: Education, Type 2 diabetes, Ramadan, Fasting

\section{Introduction}

According to the International Diabetes Federation (IDF), the highest prevalence of diabetes has been reported in the Middle East and North Africa with more than 37 million people, which by 2035, will expectedly increase to 68 million. The report shows that the increase in the prevalence of diabetes in the developing countries, including Iran is significant (1). The estimated worldwide prevalence of diabetes reported the prevalence of $8.4 \%$ type 2 diabetes in Iran in 2013 that will increase to $12.3 \%$ in 2035 (1). 
The Epidemiology of Diabetes and Ramadan (EPIDIAR) study on 12243 people in 13 Muslim countries showed that $79 \%$ of people with type 2 diabetes fast during Ramadan (2). On the other hand, fasting hours may increase upto 16-20 hours a day in summers in some countries, and with the long hours of fasting, the risk of diabetes-related complications such as hypoglycemia, hyperglycemia, diabetic ketoacidosis, dehydration and thrombosis increases (2). The EPIDIAR study showed 3 and 5-fold increases of hyperglycemia admission during Ramadan in patients with types 1 and 2 diabetes, respectively. Also the incidence of hypoglycemia in patients with type 2 diabetes and hospitalization due to hypoglycemia in patients with types 1 and 2 diabetes increases 7.4 and 5.7-fold, respectively (2).

Evidence suggests that adverse effects of Ramadan fasting may be prevented by training (3-5), adjusting the type and dose of medication and appropriate lifestyle changes (3). Previous studies have shown the effects of Ramadan educational programs on the patients' ability to change their lifestyle (6-8). A comprehensive training program can reduce the risk of these complications, weight management and metabolic control during the month of Ramadan (9). HBM is one of the most common models that is used in the practical programs of health behaviors (10-14). This model emphasizes the fact that individual perceptions and beliefs about the fear of health problems and assessing the benefits and barriers to healthy behaviors make the people behave in a different way (15).

Diabetes is a chronic condition and the patients affected by it should administer the treatment themselves; therefore, in order to prevent or reduce diabetes complications, it seems necessary to educate these patients how to control their blood sugars. It seems that the HBM can be useful as the base of the researches that study the effects of different interventions on the health knowledge and belief, following diabetic diet and diabetes treatment because misunderstanding about this disease can lead to some unpleasant changes in the patients' lifestyle (16). Considering the above issues and the importance of education in controlling diabetes, this study was conducted in order to study the effects of educational programs based on HBM for the people affected by type 2 diabetes who fast during Ramadan.

\section{Materials and Methods}

This research was a randomized control trial involving control and intervention groups. The participants were recruited from among 30 to 60 -yearold patients suffering from type 2 diabetes for at least 3 years, visiting the Endocrinology and Metabolism Research Center of Tehran University of Medical Sciences in 2015, being treated with oral hypoglycemic drugs, tending to go on fast during Ramadan and have been fasting for at least 15 days in the last years. Among these patients, those with acute illnesses during fasting, history of severe and frequent hypoglycemia without any reason during 3 months before Ramadan, without warning symptoms of hypoglycemia, history of diabetic ketoacidosis (DKA) or hyperosmolar hyperglycemic coma during 3 months before Ramadan, suffering from advanced macro-vascular complications or the side effects aggravating diabetes symptoms, pregnancy, AIC hemoglobin more than 9, insulin therapy, and changes in lipid and blood pressure drugs during Ramadan were not selected as participants.

Sample size was calculated according to the mean change in the frequency of hypoglycemia episodes during the month of Ramadan in the two study groups. Considering the power of $90 \%, Z_{\beta}=1.28$, confidence level of $95 \%$, and $\mathrm{Z}_{\alpha / 2}=1.96,21$ patients were determined for each group. With the possibility of the sample loss of $15 \%$, totally, 50 people with type 2 diabetes were included in the study. They were randomly assigned into two equal groups based on random block procedure and the table of random numbers. Both groups were received the routine public knowledge. Besides, the intervention group received a training program. All of the subjects had adequate literacy. The patients were asked to consult with their physician about changing their drug doses before Ramadan. The drugs should be prescribed according to standard recommendations. After describing the research goals, they were invited to participate in the research. Then the patients signed a consent form for participating in the this project, which was approved by the Ethics Committee of the Institute of Endocrinology and Metabolism of Tehran University of Medical Sciences and was coded and registered as EC-00368 in the Iranian Registry of Clinical Trials (IRCT). Those who fasted less than 15 days, not attended the required sessions, not 
completed their dietary records (with no self measurement of blood glucose), and those experienced symptoms of severe hypoglycemia or hyperglycemia during the study were excluded from the study.

The data were collected using a questionnaire by a trained nutritionist; then they were anonymously coded and ordered in three parts of demographic information, HBM aspects, and assessment of knowledge structures. Dietary intakes were assessed using a 24-h recall questionnaire, and the obtained data were analyzed for total calorie intake and macronutrients using the adjusted N4 software (Nutritionist: version 4.0; Tinuviel Software, Warrington, United Kingdom). Height was measured with a wall-mounted stadiometer to the nearest 0.1 $\mathrm{cm}$. Weight was determined to the nearest $0.1 \mathrm{~kg}$ on the same properly calibrated electronic digital scale, without shoes, with minimal clothing, and after voiding. Body mass index (BMI) was estimated as the ratio of body weight to height squared, and was expressed as $\mathrm{kg} / \mathrm{m}^{2}$. Waist circumference was determined by placing a measuring tape in a horizontal plane around the abdomen just above the right iliac crest. Physical activity level was assessed by a validated questionnaire in which nine different metabolic equivalent (MET) levels were ranged on a scale from sleep/rest (0.9 METs) to high-intensity physical activity (> 6 METs).

In assessing the structures of HBM, the questionnaire including 21 questions was designed using similar studies done on applying HBM in educating the patients with diabetes $(10,12)$ and the results obtained from the studies done on educating how to fast in diabetic patients (17). This questionnaire also includes 4 questions as to the perceived susceptibility to fasting during Ramadan, 3 questions as to the perceived severity, 3 questions as to the perceived benefits, 4 questions as to the perceived barriers, 2 questions as to guidelines, and 5 questions as to the perceived self-efficacy. The questionnaire was designed according to Likert scale. Each question has 5 choices, and the scores range from 1 to 5 . The choices were quite agree, agree, no idea, disagree, and totally disagree, which were scored from 5 to 1 , respectively. The validity of the data collection tools was assessed using Content Validity. The initial questionnaire designed based on
HBM was given to some health education specialists, endocrinologists, and nutritionists to correct the errors. The reliability of the questionnaire was evaluated using test-retest method. To do so, the questionnaire was completed by the researcher for 10 people with type 2 diabetes, who were demographically similar to those participating in the study and wanted to go on fast. After two weeks, the questionnaire was completed by the researcher on the same group in the same conditions; then the Cronbach's alpha coefficient was calculated for every structure in the pilot study. This coefficient for perceived susceptibility, severity, benefits, barriers, self-efficacy and guideline was obtained as $0.75,0.69$, $0.74,0.70,0.66$ and 0.71 , respectively. The subjects participating in the pilot study were excluded from the main study. For the main study, the questionnaire was finally edited.

In the third part, a questionnaire on "studying the awareness of diabetic patients in terms of fasting and its effective factors" was used whose reliability and validity had been confirmed before (17). In order to design this questionnaire, first the concepts and issues were designed based on previous studies and interviews with diabetic patients in the form of multiple choice questions. The face validity of the questionnaire was first assessed by the specialists, and the errors were corrected. The content validity was also carefully considered, and the necessary changes were made in the questionnaire. In a pilot study, the modified version of the questionnaire was completed by the researcher for 20 diabetic patients, who had been selected through simple sampling, and its reliability was tested based on the Chronbach's alpha coefficient that was equal to 0.79 . The final questionnaire included 29 questions in 5 main categories- 6 questions regarding fasting conditions for diabetic patients and how to take the blood sugar controlling drugs during Ramadan, 5 questions regarding how to control the blood sugar, and 18 questions regarding the nutrition and physical activity of diabetic patients during Ramadan. Each answer to these questions was considered as one point, and the final score of each person was expressed as a percentage (17).

One week before Ramadan, the patients in the intervention group were educated based on HBM. The course was run during 4 days each one for two hours. 
The educational program was designed and developed according to the principles of diabetes management during Ramadan established by the ADA (American Diabetes Association) (8), the results obtained from the conducted interviews, the information collected from the previous studies on the same group (17), other findings of the conducted studies on educating the fasting diabetic patients $(3,18)$, and the recommendations offered by medical professionals in terms of HBM. The syllabus included diabetes and fasting physiology, fasting conditions for diabetic patients, signs and symptoms related to diabetes and fasting (like hypoglycemia and hyperglycemia), the times to control the blood sugar during fasting and break the fast, how to set up a healthy and balanced diet that provides enough energy and nutrients, minimizing the foods containing high amounts of fats and sweets, using nutrients to prevent hypoglycemia and dehydration during hours, choosing the right food when breaking the fast, the type, level and time of physical activity, and how to take drugs during Ramadan. The training sessions were held through lectures and group discussions. At the end of each session, some pamphlets containing information about the related subject were distributed among the participants. The control group was not educated, but after completing the questionnaires, they received the same pamphlets.
Statistical analysis: The data were analyzed using SPSS 16 (Version 16, SPSS Inc., Chicago, IL). The information related to Kolmogorov-Smirnov's test was used to evaluate the normality of the quantitative variables. The quantitative variables of the intervention and control groups were compared using the independent t-test or Mann-Whitney's U test. Also the qualitative variables were compared using Chisquared test and Fisher's exact test. Moreover, the statistical tests, independent t-test and paired t-test were used in order to study the significance. The level of significance was considered as $P<0.05$.

\section{Results}

Seven out of the 50 people participating in this study were excluded from the control group because they got hypoglycemia and did not want to fast anymore, and the data derived from 25 people in the intervention group and 18 people in the control group were analyzed.

In Table 1, the frequency distribution of demographic features, and in Table 2, the frequency distribution of the lifestyle and anthropometric features of the people in both groups are shown. Comparing the data of the people remaining in the study with the data related to the excluded ones did not show any significant statistical differences.

Table 1. Frequency distribution of the demographic features of the people in the intervention and control groups at the beginning of the study*

\begin{tabular}{|c|c|c|c|c|}
\hline Variables & & $\begin{array}{c}\text { Intervention group } \\
(\mathrm{N}=25)\end{array}$ & $\begin{array}{l}\text { Control group } \\
(\mathrm{N}=25)\end{array}$ & $P$-value ${ }^{+\dagger}$ \\
\hline Age (Yrs) & & $53.41 \pm 8.59$ & $53.33 \pm 6.20$ & 0.975 \\
\hline \multirow[t]{2}{*}{ Sex } & Woman & $13(52.0 \%)$ & $8(32 \%)$ & \multirow[t]{2}{*}{0.114} \\
\hline & Man & $12(48.0 \%)$ & $17(68.0 \%)$ & \\
\hline Duration of diabetes & & $6.8 \pm 6.2$ & $7.0 \pm 5.8$ & 0.927 \\
\hline \multirow[t]{5}{*}{ Level of education } & Illiterate & $2(8.0 \%)$ & $1(4 \%)$ & \multirow[t]{5}{*}{0.158} \\
\hline & Primary school & $1(4.0 \%)$ & $2(8 \%)$ & \\
\hline & Guidance school & $3(12.0 \%)$ & $1(4 \%)$ & \\
\hline & High school & $10(40.0 \%)$ & $8(32 \%)$ & \\
\hline & University & $9(36.0 \%)$ & $13(52 \%)$ & \\
\hline \multirow[t]{2}{*}{ Marital status } & Single & $2(8.0 \%)$ & $3(12.0 \%)$ & \multirow[t]{2}{*}{0.445} \\
\hline & Married & $23(92.0 \%)$ & $22(88.0 \%)$ & \\
\hline
\end{tabular}

\footnotetext{
* The quantitative and qualitative variables were reported as mean \pm standard deviation and number (percentage) respectively.
}

Quantitative variables were compared using independent t-test or Mann-Witney's and qualitative variables were compared using Chi-squared test and Fisher's exact test. 
The effect of education on different aspects of HBM: Table 3 shows the mean and standard deviation of the score of different aspects of HBM before and after Ramadan in both groups. As it is clear, education significantly increases the perceived severity, perceived benefits, perceived barriers and self-efficacy in comparison with the basic values $(\mathrm{P}<$ 0.001 ) and also compared with the control group $(\mathrm{P}=0.003, \mathrm{P}=0.001, \mathrm{P}=0.002$ and $\mathrm{P}<0.001$ for the mean of changes in the scores of perceived severity, perceived benefits, perceived barriers and selfefficacy in the control and intervention groups, respectively). The score of perceived benefits in the control group increased significantly ( $\mathrm{P}=0.011)$.

The effect of education on different aspects of knowledge: Table 4 shows the mean and standard deviation of the scores of different aspects knowledge, including the fasting and drug use conditions, controlling blood sugar, nutrition and physical activity before and after Ramadan in the control and intervention groups. There was not a significant statistical difference in any of the knowledge aspects as well as in the total score of knowledge between the two groups at the beginning of the study. At the end of the study, there was a significant statistical difference in the score of fasting and drug use conditions, nutrition and physical activity and the total score of knowledge between the two groups $(\mathrm{P}<0.001, \quad \mathrm{P}=0.049$ and $\mathrm{P}=0.013$, respectively).

According to the findings, education significantly increased the scores related to fasting and drug use conditions, controlling blood sugar, and the total score of knowledge in comparison with the basic values $(\mathrm{P}=0.002, \mathrm{P}=0.026$ and $\mathrm{P}=0.039)$. In addition, the educational program significantly increased the scores related to fasting and drug use conditions, controlling blood sugar, and the total score of knowledge in comparison with the control group ( $\mathrm{P}=0.011, \mathrm{P}=0.016$ and 0.045$)$.

Table 2. Comparison of the frequency distribution of the lifestyle and anthropometric features of the people in the intervention and control groups at the beginning of the study ${ }^{*}$

\begin{tabular}{lccc}
\hline Variables & & $\begin{array}{c}\text { Intervention group } \\
(\mathrm{N}=25)\end{array}$ & Control group (N=25) \\
\hline Smoking & Yes & $1(4.0 \%)$ & $1(4 \%)$ \\
& No & $22(86.0 \%)$ & $20(80 \%)$ \\
Energy intake (Kcal per day) & & $1935 \pm 466$ & $2043 \pm 693$ \\
Macronutrients & Carbohydrate & $276.90 \pm 84.99$ & $238.68 \pm 84.82$ \\
(Gram per day) & Protein & $73.26 \pm 21.97$ & $74.91 \pm 27.93$ \\
& Lipid & $69.62 \pm 25.17$ & $81.33 \pm 45.50$ \\
Physical activity & & $1.54 \pm 0.16$ & $1.49 \pm 0.15$ \\
BMI $\left(\mathrm{Kg} / \mathrm{m}^{2}\right)$ & & $29.20 \pm 4.51$ & $28.17 \pm 4.11$ \\
Waist circumference $(\mathrm{cm})$ & & $100.50 \pm 10.12$ & $98.73 \pm 10.58$ \\
\hline
\end{tabular}

* The quantitative and qualitative variables were reported as mean \pm standard deviation and number (percentage), respectively.

Quantitative variables were compared using independent t-test or Mann-Witney's test, and qualitative variables were compared using Chi-squared test and Fisher's exact test.

Table 3. Comparison of the different aspects of HBM before and after intervention

\begin{tabular}{lccc}
\hline Variables & $\begin{array}{c}\text { Intervention group } \\
(\mathrm{N}=25)\end{array}$ & $\begin{array}{c}\text { Control group } \\
(\mathrm{N}=18)\end{array}$ & Net difference for the change \\
\hline Perceived susceptibility & $-0.33 \pm 1.11$ & $-0.20 \pm 0.41$ & $0.13 \pm 0.7$ \\
Perceived severity & $-2.86 \pm 1.72^{\dagger}$ & $-0.87 \pm 1.60$ & $1.99 \pm 0.12^{\psi}$ \\
Perceived benefits & $-3.27 \pm 1.71^{\dagger}$ & $-1.13 \pm 1.50^{\dagger}$ & $2.14 \pm 0.21^{\psi}$ \\
Perceived barriers & $2.33 \pm 1.11^{\dagger}$ & $-0.20 \pm 0.41$ & $2.53 \pm 0.7^{\psi}$ \\
Self-efficacy & $-7.87 \pm 4.99^{\dagger}$ & $-0.47 \pm 1.12$ & $7.4 \pm 3.87^{\varphi}$ \\
\hline
\end{tabular}

${ }^{\dagger} P<0.001$ within group changes

${ }^{\psi} P<0.005,{ }^{\varphi} P<0.001$ between-group differences for the changes 
Table 4. Comparing different aspects of knowledge before and after intervention

\begin{tabular}{lccc}
\hline \multicolumn{1}{c}{ Variables } & $\begin{array}{c}\text { Intervention } \\
\text { group }(\mathrm{N}=25)\end{array}$ & $\begin{array}{c}\text { Control group } \\
(\mathrm{N}=18\end{array}$ & $\begin{array}{c}\text { Net difference for } \\
\text { the change }\end{array}$ \\
\hline Fasting and drug use conditions & $-1.18 \pm 1.80^{\dagger}$ & $-0.17 \pm 0.71$ & $1.01 \pm 1.09^{\varphi}$ \\
Controlling blood sugar & $-1.03 \pm 1.29^{\psi}$ & $0.05 \pm 1.66$ & $1.08 \pm 0.37^{\varphi}$ \\
Nutrition and physical activity & $-0.93 \pm 5.14$ & $-0.33 \pm 0.48^{\psi}$ & $0.6 \pm 4.66$ \\
Total score of knowledge & $-2.75 \pm 3.37^{\psi}$ & $-0.72 \pm 1.18$ & $2.03 \pm 2.19^{\varphi}$ \\
\hline${ }^{\dagger} P<0.005,{ }^{\psi} P<0.05$ within group changes & & &
\end{tabular}

\section{Discussion}

The educational program executed in the intervention group made the perceived severity, perceived benefits, perceived barriers and selfefficacy to increase significantly in comparison with the basic values and also the control group. At the end of the study, there was a significant statistical difference in the scores related to the knowledge in terms of fasting and drug use conditions, nutrition and physical activity between the two groups. The educational program executed in the intervention group made the scores related to fasting and drug use conditions, controlling blood pressure, and the total score of knowledge increase significantly in comparison with the basic values and the control group.

Fasting during Ramadan makes a lot of changes in the metabolism, habits and lifestyle, including avoiding food and drink from dawn to sunset, especially during hot months of the year, increasing the variety of foods (particularly sugary and bulky foods for breaking the fast), changing the quantity and time of taking drugs and changing the sleep patterns and physical activity $(17,8)$ that can lead to increase the risk of hypoglycemia and hyperglycemia (2). The advantages and disadvantages of fasting in patients with type 2 diabetes are still under consideration. Despite the conflicting results of some studies (19), the results show that fasting during Ramadan can lead to increase the risk of hypoglycemia and hyperglycemia in the patients with type 1 and 2 diabetes (2).

Some studies have emphasized on the role of selfcaring in controlling the diabetes. The patients who are not able to care for themselves cannot set off a normal reaction against hypoglycemia and hyperglycemia (20). Therefore, it is necessary for diabetic patients to be able to care for themselves and make more effort to their blood sugar (8).

Currently, many physicians believe that fasting during Ramadan can be acceptable for the patients who control their diabetes properly, provided that they are aware of health conditions, and lifestyle choices (i.e. diet and drug, and sport) (21). However, the researches that studied the knowledge of patients about fasting suggest that they have very limited knowledge $(17,18)$. The researches done in both the developing and developed countries show that limited knowledge makes diabetic patients not to follow medical recommendations and orders dutifully (22).

The benefits and advantages of education for diabetic patients have been proven in different studies $(23,24)$. The value of an educational program depends on its effectiveness in improving the health behaviors, which is not easily achievable. The effectiveness of education depends on the appropriate use of behavioral science theories that have great potential for increasing the effectiveness of health education programs; therefore, it is very important to develop and run these programs based on theories. HBM is a model that is used to learn how to have a diet (25). These educational programs should have four essential features to be effective in improving the patients' behavior: first, the health behavior should be advantageous in terms of economy, society, culture and family; second, the educators should identify the personal, social, economic, cultural and family barriers to adopt certain health behaviors and plan based on them; third, the educational programs should be able to warn the patients against the complications resulting from the lack of health behaviors; and fourth, in order to adopt certain health behaviors, it is necessary to use other guides such as recommendations of health care staffs and physicians, mass media, posters, pamphlets and reminder cards (25).

The present study focused on the effects of education on fasting diabetic patients based on HBM while many other studies have considered the effect of education on diabetic patients. These researches have shown that the structures of HBM, including susceptibility, severity, benefits, barriers and self- 
efficacy are improved after running the educational programs $(13,26-31)$. This research showed that there is a significant difference between the scores related to the perceived severity, perceived benefits, perceived barriers and self-efficacy in comparison with the basic values and also with the control group. Along with this significant increase in the scores related to the structures of HBM of the patients in the intervention group, Zinat Motlagh et al. (32) and Sharifirad et al. (33) also found a significant increase in the scores related to perceived severity, perceived benefits, and self-efficacy and a decrease in the perceived barriers in comparison with the basic values and also the control group $(\mathrm{P}<0.005$ and $\mathrm{P}<0.001$, respectively). In addition, the mean of nutrition knowledge score related to the patients in the intervention group increased significantly $(\mathrm{P}<0.050$ and $\mathrm{P}<0.001$, respectively). In a research studying the effects of education using HBM, Asadi Zandi et al. (12) reported an increase in the scores of the structures, including the perceived susceptibility, perceived severity, and self-efficacy, and the total score of self-caring $(\mathrm{P}<0.005)$, but they showed that the scores related to the perceived benefits and barriers are not changed significantly. Also Sadeghi et al. reported an improvement in the scores of the perceived benefits, perceived barriers and guidelines in the intervention group. After the educational program, the total score of knowledge in the intervention group was significantly different from that in the control group $(12,14)$.

Promoting the awareness of patients in the intervention group of the present study is similar to that resulted from another study, which was conducted for educating diabetic patients on how to fast. In the research done by McEwen et al., for example, 515 diabetic patients from Egypt, Iran, Lebanon, Saudi Arabia and the U.S. were selected as samples to be educated. After running the educational program, the knowledge of these patients regarding the signs and symptoms of hypoglycemia increased in comparison with 259 other diabetic patients who did not receive such education $(0.16 \pm 1.2$ vs. $-0.14 \pm 1.1$, $\mathrm{P}=0.007)$. In another study, Masood et al. also showed that the patients who received education from physicians, diabetes and diet educators are more knowledgeable about the principles of controlling diabetes during Ramadan, like the signs and symptoms of hypoglycemia and hyperglycemia, drugs and insulin dosage during and after Ramadan, controlling the sugar blood, nutritional information, physical activity, and food and drink intake during Ramadan than those who did not receive such education $(\mathrm{P}<0.05)(4)$. Another research conducted on Indian patients with type 2 diabetes indicated that, after training, the knowledge of the the participants about the lifestyle, diet and drug increased significantly $(\mathrm{P}<0.05) \quad(34)$.

Regarding the key role the patients play in controlling and treating their diabetes and considering the results of the studies that were done based on the possibility of controlling and preventing the shortterm and long-term symptoms of diabetes, it seems that education has a crucial role in patients' coping with this disease. On the other hand, the value of an educational program depends on its effectiveness in the appropriate use of theories and models that are used in health education. The educational programs are developing from just education into engaging the patients with the treatment process, focusing on the patients' self-efficacy and empowerment through psychological methods, such as dealing with false beliefs, modifying emotional reactions to diabetes, and finally, interventioning based on personal model.

One of the main advantages of the present study is using the HBM as the most useful behavioral science theory that increases the effectiveness of educational intervention. Moreover, in this research, valid questionnaires were used to assess the knowledge of fasting in diabetic patients. Collecting data and running educational programs were done by a group of experts including health education specialists, endocrinologists, and nutritionists to reduce the possibility of information bias.

In dealing with the findings of the research, it is necessary to consider the limitations of the study. One of these limitations is that the number of the patients participating as the sample in this research is few. On the other hand, at the beginning of the research, some patients might try more to control blood sugar and avoid its changes during Ramadan; this might cause some bias in selecting the samples. Also fasting cessation as a result of hypoglycemia in the significant proportion of the control group may lead to making to some extent unreliable conclusions.

\section{Conclusion}

The results obtained from this research showed that the educational programs executed in the 
intervention group increased their level of knowledge and improved the structures of the HBM in comparison with the control one. The effort to provide the patients with education about fasting requires the diabetes communities, health care professionals, religious and social leaders and media to take a multipronged approach. However, there is little data on studying educational intervention for fasting diabetics. The authors suggest that some studies should be done with more samples on a larger scale to find better ways to support fasting diabetics.

\section{Acknowledgement}

This study was funded by the Endicrinology and Metaboloism Research Institiute of Tehran University of Medical Sciences, Tehran, Iran. The authors thank the staffs of Diabetes Clinic of the Endicrinology and Metaboloism Research Institiute for helping in recruiting patients.

\section{Financial disclosure}

The authors declared no financial interest.

\section{Funding/Support}

This work was financially supported by the Endicrinology and Metaboloism Research Institiute of Tehran University of Medical Sciences, Tehran, Iran.

\section{References}

1. Guariguata L, Whiting D, Hambleton I, Beagley J, Linnenkamp U, Shaw J. Global estimates of diabetes prevalence for 2013 and projections for 2035. Diabetes Research and Clinical Practice. 2014;103(2):137-49.

2. Salti I, Bénard E, Detournay B, Bianchi-Biscay M, Le Brigand C, Voinet C, et al. A Population-Based Study of Diabetes and Its Characteristics During the Fasting Month of Ramadan in 13 Countries Results of the Epidemiology of Diabetes and Ramadan 1422/2001 (EPIDIAR) study. Diabetes Care. 2004;27(10):2306-11.

3. Bravis V, Hui E, Salih S, Mehar S, Hassanein M, Devendra D. Ramadan Education and Awareness in Diabetes (READ) programme for Muslims with Type 2 diabetes who fast during Ramadan. Diabetic Medicine. 2010;27(3):327-31.

4. Masood SN, Alvi SFD, Ahmedani MY, Kiran S, Zeeshan NF, Basit A, et al. Comparison of Ramadanspecific education level in patients with diabetes seen at a Primary and a Tertiary care center of KarachiPakistan. Diabetes \& Metabolic Syndrome: Clinical Research \& Reviews. 2014;8(4):225-9.

5. McEwen LN, Ibrahim M, Ali NM, Assaad-Khalil SH, Tantawi HR, Nasr G, et al. Impact of an individualized type 2 diabetes education program on clinical outcomes during Ramadan. BMJ open diabetes research \& care. 2015;3(1):e000111.
6. Steinsbekk A, Rygg L, Lisulo M, Rise MB, Fretheim A. Group based diabetes self-management education compared to routine treatment for people with type 2 diabetes mellitus. A systematic review with metaanalysis. BMC Health Services Research. 2012;12(1):213.

7. Chrvala CA, Sherr D, Lipman RD. Diabetes selfmanagement education for adults with type 2 diabetes mellitus: A systematic review of the effect on glycemic control. Patient Education and Counseling. 2015.

8. Al-Arouj M, Assaad-Khalil S, Buse J, Fahdil I, Fahmy M, Hafez S, et al. Recommendations for management of diabetes during Ramadan update 2010. Diabetes Care. 2010;33(8):1895-902.

9. Al-Amoudi A, Al-Ulagi N, Bashir M, Bissar L, AlGhamdi S, Elhadd T. Education for diabetic patients for fasting of Ramadan: a questionnaire study. 2006; P277.

10. Baghiani Moghadam M, Taheri G, Fallah Zadeh H, Parsa M. The effect of instructional designed SMS based on Health Belief Model (HBM) on adoption of self-care behavior of patients with type II diabetes. Modern Care Journal. 2014; 11 (1) :10-18.

11. azini H, Barati M. Predicting Factors Related to Selfcare Behaviors among Type 2 Diabetic Patients based on Health Belief Model. Journal of Torbat Heydariyeh University of Medical Sciences 2014;1(4):16-25.

12. Asadzandi M, Farsi Z, Najafi Mehri S, Karimizarchi A A. Educational intervention focusing on health beliefe model in health beliefs, awareness and behavior of diabetic patients. IJDLD. 2006; 6 (2) :169-176.

13. Sharifirad G, Hazavehie S, Mohebi S, Rahimi M, Hasanzadeh A. The effect of educational programme based on Health Belief Model (HBM) on the foot care by type II diabetic patients. Iranian Journal of Endocrinology and Metabolism. 2006; 8 (3) :231-239.

14. Sadeghi R, Rezaeian M, Khanjani N, Iranpour A. The Applied of Health Belief Model in Knowledge, Attitude and Practice in People Referred for Diabetes Screening Program: An Educational Trial. Journal of Rafsanjan University of Medical Sciences. 2015; 13 (11):10611072.

15. Saffari M, Shojaeizadeh D, ghafranipoor F, Heydarnia A. Theories, models and methods of health education and health promotion. Sobhan Press 2009: 53-63.

16. Becker MH, Janz NK. The health belief model applied to understanding diabetes regimen compliance. The Diabetes Educator. 1985;11(1):41-7.

17. khoundan M, Shadman Z, Poorsoltan N, Soleymanzadeh M, Khoshniat Nikoo M, Larijani B. Designing a questionnaire to evaluate diabetic patients' knowledge of ramadan fasting and its determinant factors. IJDLD. 2014; 13 (4):331-339.

18. Gaborit B, Dutour O, Ronsin O, Atlan C, Darmon P, Gharsalli R, et al. Ramadan fasting with diabetes: an interview study of inpatients' and general practitioners' 
attitudes in the South of France. Diabetes \& Metabolism. 2011;37(5):395-402.

19. Bakiner O, Ertorer ME, Bozkirli E, Tutuncu NB, Demirag NG. Repaglinide plus single-dose insulin glargine: a safe regimen for low-risk type 2 diabetic patients who insist on fasting in Ramadan. Acta Diabetologica. 2009;46(1):63-5.

20. Norris SL, Engelgau MM, Narayan KV. Effectiveness of self-management training in type 2 diabetes a systematic review of randomized controlled trials. Diabetes Care. 2001;24(3):561-87.

21. Farid D, Rosenberg E, Bartlett G. Importance of education in managing type 2 diabetes during Ramadan. Canadian Family Physician. 2014;60(6):508-10.

22. Badruddin N, Basit A, Hydrie MZI, Hakeem R. Knowledge, attitude and practices of patients visiting a diabetes care unit. Pakistan Journal of Nutrition. 2002;1(2):99-102.

23. Norris SL, Lau J, Smith SJ, Schmid CH, Engelgau MM. Self-Management education for adults with type 2 Diabetes A meta-analysis of the effect on glycemic control. Diabetes Care. 2002;25(7):1159-71.

24. Ellis SE, Speroff T, Dittus RS, Brown A, Pichert JW, Elasy TA. Diabetes patient education: a meta-analysis and meta-regression. Patient Education and Counseling. 2004;52(1):97-105.

25. Sharifi Rad GH, Entezari Mohammad H, Aziz K, Azadbakht L. The effectiveness of nutrition education for patients with type 2 diabetes: Health Belief Model. Iranian Journal of Endocrinology and Metabolism. 1999; 7(4):379-386.

26. Bayat F, Shojaeezadeh D, Baikpour M, Heshmat R, Baikpour M, Hosseini M. The effects of education based on extended health belief model in type 2 diabetic patients: a randomized controlled trial. J Diabetes Metab Disord. 2013;12(1):45.
27. Jalilian F, Motlagh FZ, Solhi M, Gharibnavaz H. Effectiveness of self-management promotion educational program among diabetic patients based on health belief model. Journal of Education and Health Promotion. 2014;3.

28. AGHA MT, Eftekhar H, Mohammad K. Application of health belief model to behavior change of diabetic patients. 2005.

29. Shamsi M, Hassanzadeh A, Kachoyee A, Sharifirad G. Influence of walking training on haemoglobin glucosile and fasting blood sugar levels in women with type 2 diabetes. Koomesh. 2010; 11 (2) :99-106.

30. Hiedarnia AR, Rakhshani F, Zareban I, Moshki M , Sharakipour M, Niknami SH. The effect of education based on health belief model on reduction of HbA1c level in diabetestype 2 . Journal of Research \& Health. 2013; 3(2) : 370-378.

31. Mardani M, Vahed Aziz SH. The effect of education based on HBM on adherence to diabetic diet. Iranian Journal of Endocrinology and Metabolism. 2010;9(3): 268-275.

32. Zinat Motlagh F, Sharifirad GH, Jalilian F, Mirzaei Alavijeh M, Aghaei A, Ahmadi-Jouibari T. Effectiveness of Educational Programs to Promote Nutritional Knowledge in Type II Diabetes Patients Based on Health Belief Model. J Health Syst Res 2013; 9(4): 412-20.

33. Sharifirad G, Entezari MH, Kamran A, Azadbakht L. The effectiveness of nutritional education on the knowledge of diabetic patients using the health belief model. Journal of research in medical sciences: the official journal of Isfahan University of Medical Sciences. 2009;14(1):1.

34. Fatim J, Karoli R, Chandra A, Naqvi N. Attitudinal determinants of fasting in type 2 diabetes mellitus patients during Ramadan. J Assoc Physicians India. 2011;59:630-4. 